\title{
Laborreform der Stufe 2 in Berlin
}

\author{
Christoph Sucker Günther Kappert
}

Hämostaseologie 2020;40:133.

Begründet dadurch, dass die Laborreform der Stufe 1 nicht zu einer Senkung der Laborkosten führte, treten im Bereich der kassenärztlichen Vereinigung (KV) Berlin zum 01.01.2020 Neuerungen in Kraft, die die Kosten von Laboruntersuchungen konstant halten bzw. reduzieren sollen.

Konkret wird in Berlin zum 01.01.2020 die Vergütung der auf Überweisungsschein 10 veranlassten Laborleistungen der Abschnitte 32.2 und 32.3 des einheitlichen Bewertungsmaßstabes (EBM) begrenzt. Hierzu werden sogenannte praxisindividuelle Laborbudgets (piLab) eingerichtet, deren Höhe sich aus dem im Zeitraum vom zweiten Quartal 2017 bis zum ersten Quartal 2018 durchgeführten Laborleistungen der jeweiligen Einrichtung ergibt; es werden somit die entsprechenden Praxis umsetzbar aus den letzten 4 Quartal vor Durchführung der Laborreform der Stufe 1 zugrunde gelegt. Konkret bedeutet dies, dass die Leistungen der auf Überweisungsschein 10 durchgeführten Laboruntersuchungen entsprechend der eingeführten Laborquote mit $89 \%$ des ausgewiesenen EBM-Betrages erstattet werden; bei Überschreitung des piLab werden darüber hinaus durchgeführte Laborleistungen nur noch mit 33 \% erstattet. Es ist vorgesehen, dass piLab mit einer Dynamisierungsrate von ca. zusätzlich 4-8\% quartalsweise fort zu entwickeln. Wenngleich die Laborreform der Stufe 2 in dieser Form zunächst nur im Gebiet der kassenärztlichen Vereinigung (KV) Berlin umgesetzt wird, ist dennoch zu erwarten, dass diese Reform auch auf andere KV-Regionen ausgeweitet wird.

Was bedeutet dies konkret für Einrichtungen, die Laborleistungen der EBM-Kapitel 32.2 und 32.3 auf Überweisungsschein 10 durchführen? Für diese Einrichtungen wird der Umsatz entsprechender Laborleistungen mit einer gewissen Dynamisierung auf die zugrunde gelegten Basis Quartal oder 02/2017-0 1/2018 quasi eingefroren. Dies ist insbesondere für solche Einrichtungen dramatisch, die im Jahr 2019 eine erhebliche Zunahme des Umsatzes genau in diesem Bereich realisiert haben; für diese bedeutet das, dass ihre zusätzlichen Leistungen weitaus schlechter vergütet werden, nämlich nur mit einem Drittel des ausgewiesenen EBMWertes. Es liegt auf der Hand, dass gerade die Erbringung von Leistungen, bei denen nur eine geringe Marge erzielt werden kann, für diese Laboratorien kontraproduktiv sind, da sie nicht zu einem vermehrten Gewinn, sondern gar zu einem Verlust führen. Des Weiteren ist problematisch, dass die Einsender von Laborüberweisungen über Überweisungsschein Muster 10 natürlich weiter entsprechende Proben an ihre Partnerlabore versenden werden, die dann in der Regel diese Untersuchungen nicht zurückweisen dürfen; aus Sicht der kassenärztlichen Vereinigung sollen die Laboratorien darauf hinwirken, dass ihrer Einsender daher die Laborleistungen mit noch mehr Sorgfalt als bisher anfordern, um Kosten zu sparen. In der Praxis dürfte sich dies als äußerst schwierig umsetzbar erweisen. Es steht daher zu befürchten, dass die Laboratorien Laborleistungen, die dann nicht mehr kostendecken erbracht werden können, aus ihren Portfolio ausstreichen und outsourcen. Dies wiederum kann zu personellen Konsequenzen in den entsprechenden Einrichtungen mit Kündigung von Ärzten und Laborpersonal führen. Möglicherweise werden die Einrichtungen, die Laboruntersuchungen im Rahmen der Mit- und Weiterbehandlung von Patienten auf einen Überweisungsschein Nummer 6 erbringen, durch die getroffenen Maßnahmen eine Zunahme der Patientenzahlen erfahren, da die Erbringung entsprechender Leistungen durch Probeneinsendung mit Überweisungsschein Muster 10 für die entsprechenden Laboratorien nicht mehr attraktiv ist. Dies wiederum könnte zu einer weiteren Überlastung der ohnehin schon stark frequentierten Gerinnungsambulanzen führen. Die weitere Entwicklung bleibt abzuwarten. Bei neuen Entwicklungen werden wir an dieser Stelle gerne wieder berichten.

Einladung zur Mitgliederversammlung des BDDH e.V. im Rahmen der GTH e.V.:

Termin: Donnerstag, 20.02.2020, 8.15 Uhr - 9.45 Uhr, Raum Kaisen

Thema: Mitgliederversammlung mit Wahl des Vorstands

Für den Vorstand des Berufsverbandes der Deutschen Hämostaseologen e.V. (BDDH):

Priv.-Doz. Dr. med. habil. Christoph Sucker, Berlin

2. Beisitzer des Vorstands

Dr. med. Günther Kappert, Duisburg

1. Beisitzer des Vorstands
(C) 2020 Georg Thieme Verlag KG Stuttgart · New York
DOI https://doi.org/ $10.1055 / \mathrm{s}-0040-1701472$. ISSN 0720-9355. 